\title{
ON POLETSKY THEORY OF DISCS IN COMPACT MANIFOLDS
}

\author{
UROŠ KUZMAN
}

\begin{abstract}
We provide a direct construction of Poletsky discs via local arc approximation and a Runge-type theorem by A. Gournay [5].
\end{abstract}

Let $\mathbb{D}=\{\zeta \in \mathbb{C}:|\zeta|<1\}$ denote the open unit disc. Given a smooth (almost) complex manifold $M$ and $p \in M$, we denote by $\mathcal{O}(\overline{\mathbb{D}}, M, p)$ the set of smooth maps $u: \overline{\mathbb{D}} \rightarrow M$ that are (pseudo)holomorphic in some neighborhood of $\overline{\mathbb{D}}$ and satisfy $u(0)=p$. Given $p \in M, \epsilon>0$ and an open set $U \subset M$ we call an element of $u \in \mathcal{O}(\overline{\mathbb{D}}, M, p)$ a Poletsky disc (associated to $p, \epsilon$ and $U)$ if most of its boundary lies in $U$, t.i., there exists an exceptional set $E \subset[0,2 \pi)$ of Lebesgue measure $|E|<\epsilon$ and such that $u\left(e^{i t}\right) \in U$ for $t \notin E$. Such discs were used by E. Poletsky [11] in order to characterize the polynomial hull for compact sets in $\mathbb{C}^{n}$. Similarly, they can describe the projective hull of a compact set in complex projective spaces [2, 8].

All of the above mentioned characterizations are based on the following explicit formula for the largest plurisubharmonic minorant of a given uppersemicontinuous function $f$ on $M$ :

$$
\hat{f}(p)=\inf \left\{\int_{0}^{2 \pi} f \circ u\left(e^{i t}\right) \frac{d t}{2 \pi}: u \in \mathcal{O}(\overline{\mathbb{D}}, M, p)\right\} .
$$

The latter was proved to be valid on any complex manifold by J. P. Rosay (see [7] for related results), who also observed that if $M$ admits no nonconstant bounded plurisubharmonic function, there exists a Poletsky disc for any $p \in M, \epsilon>0$ and an open set $U \subset M$ [12, Corollary 0.2]. Indeed, in this case the minorant of the negative indicator function $f=-\chi_{U}$ equals to $\hat{f} \equiv-1$, hence the existence of the desired discs follows directly from the definition of the infimum in (1).

In this paper we present a new, direct proof of this corollary valid for a certain class of manifolds admitting a Runge-type approximation provided by A. Gournay [5]. In particular, we give a partial answer to Rosay's question raised in [12, Section 5]: given a compact complex manifold can a Poletsky

2010 Mathematics Subject Classification. 32Q60,32Q65,32E30,32U05. 
disc be provided without using (1)? Moreover, our theorem includes also some examples of almost complex manifolds.

Theorem 1. Let $M$ be a smooth, connected compact manifold equipped with a regular almost complex structure and admitting a doubly tangent property. Given a point $p \in M$, a positive constant $\epsilon>0$ and any open set $U \subset M$, there exist a disc $u \in \mathcal{O}(\overline{\mathbb{D}}, M, p)$ and a set $E \subset[0,2 \pi)$ such that $|E|<\epsilon$ and $u\left(e^{i t}\right) \in U$ for $t \notin E$.

The assumptions in the above theorem are rather technical and should be read as 'such that Gournay's approximation result applies' (we further discuss them in $\S 2$ ). However, they are fulfilled for a wide class of compact complex manifolds including complex projective spaces and Grassmannians. Moreover, as mentioned, they are valid for some manifolds equipped with a non-integrable almost complex structure $J$ (e.g. $\mathbb{C} P^{n}$ with $J$ tamed by the standard symplectic form) [5, p.313]. Note that for latter the PoletskyRosay formula (1) is proved only in a low dimensional case [6]. Hence for $\operatorname{dim}_{\mathbb{R}} M \geq 6$ and a non-integrable $J$, the theorem is new.

Finally, let us remark that in $\S 1$ we present another original statement that will be needed in the proof of the main theorem: based on [1] we provide a Mergelyan-type result for maps defined on smooth arcs (Theorem 5).

\section{The local arC Approximation}

Let $M$ be a smooth real manifold of even dimension. A $(1,1)$-tensor field $J: T M \rightarrow T M$ satisfying $J^{2}=-I d$ is called almost complex structure. A differentiable map $u:\left(M^{\prime}, J^{\prime}\right) \longrightarrow(M, J)$ between two almost complex manifolds is $\left(J^{\prime}, J\right)$-holomorphic if for every $p \in M^{\prime}$ we have

$$
J(u(p)) \circ d_{p} u=d_{p} u \circ J^{\prime}(p),
$$

We deal with two simplest cases, J-holomorphic discs $u: \mathbb{D} \rightarrow M$ and $J$ holomorphic spheres $u: \mathbb{C} P^{1} \rightarrow M$.

We denote by $J_{s t}$ the standard integrable structure on $\mathbb{C}^{n}$ for any $n \in \mathbb{N}$. In local coordinates $z \in \mathbb{R}^{2 n}$ an almost complex structure $J$ is represented by a $\mathbb{R}$-linear operator satisfying $J(z)^{2}=-I$, hence (2) equals to

$$
u_{y}=J(u) u_{x} .
$$

Further, if $J+J_{s t}$ is invertible along $u$ we have

$$
\mathcal{F}(u)=u_{\bar{\zeta}}+A(u) \overline{u_{\zeta}}=0,
$$


where $\zeta=x+i y$ and $A(z)(v)=\left(J_{s t}+J(z)\right)^{-1}\left(J(z)-J_{s t}\right)(\bar{v})$ is a complex linear endomorphism for every $z \in \mathbb{C}^{n}$. We call $A$ the complex matrix of $J$ and denote by $\mathcal{J}$ the set of all smooth structures on $\mathbb{R}^{2 n}$ satisfying the condition $\operatorname{det}\left(J+J_{s t}\right) \neq 0$.

In [1] the approximation theory was developed for the operator $\mathcal{F}$ defined as in (41) and evaluated in functions admitting a Sobolev weak derivative. In particular, given $\varphi \in W^{1, p}(\mathbb{D}), p>2$, a bounded right inverse $Q_{\varphi}$ was constructed for the derivative $d_{\varphi} \mathcal{F}$ and the following version of the Implicit Function Theorem was applied.

Theorem 2 (Implicit Function Theorem). Let $X$ and $Y$ be two Banach spaces and consider a map $\mathcal{F}: U \subset X \rightarrow Y$ of class $\mathcal{C}^{1}$ defined on an open set $U \subset X$. Let $x_{0} \in U$. Assume that the differential $d_{x_{0}} \mathcal{F}$ admits a bounded right inverse, denoted by $Q_{x_{0}}$. Fix $c_{0}>0$ such that $\|Q\| \leq c_{0}$ and $\eta>0$ such that if $\left\|x-x_{0}\right\|<\eta$ then $x \in U$ and

$$
\left\|d_{x} \mathcal{F}-d_{x_{0}} \mathcal{F}\right\| \leq \frac{1}{2 c_{0}} .
$$

Then if $\left\|\mathcal{F}\left(x_{0}\right)\right\|<\frac{\eta}{4 c_{0}}$ there exists $x \in U$ such that $\mathcal{F}(x)=0$ and

$$
\left\|x-x_{0}\right\| \leq 2 c_{0}\left\|\mathcal{F}\left(x_{0}\right)\right\| .
$$

In this paper we use the same approach to develop a similar statement for the operator $\mathcal{F}: \mathcal{C}^{1, \alpha}(\overline{\mathbb{D}}) \rightarrow \mathcal{C}^{0, \alpha}(\overline{\mathbb{D}})$. That is, we prove an analogue of [1. Theorem 5] valid for Hölder spaces (we omit the existence part for $Q_{\varphi}$ since the proof is the same as in [1, Theorem 2] and [15]). Note that these additional regularity conditions are needed since in the present paper we apply the statement to shrinking neighborhoods of an arc in order to obtain a $\mathcal{C}^{0}$-approximation. Since the diameter of such sets admits no lower bound, the $W^{1, p}$-result along with the Sobolev embedding theorem does not suffice.

Theorem 3. Let $0<\alpha<1$. Let $J \in \mathcal{J}$ and let $A$ be its complex matrix. We define $\mathcal{F}: \mathcal{C}^{1, \alpha}(\overline{\mathbb{D}}) \rightarrow \mathcal{C}^{0, \alpha}(\overline{\mathbb{D}})$ to be the operator given by

$$
\mathcal{F}(u)=u_{\bar{\zeta}}+A(u) \overline{u_{\zeta}} .
$$

For every $c_{0}>0$, there exists $\delta>0$ such that for any $\varphi \in \mathcal{C}^{1, \alpha}(\overline{\mathbb{D}})$ satisfying

$$
\|\varphi\|_{\mathcal{C}^{1, \alpha}(\overline{\mathbb{D}})} \leq c_{0}, \quad\left\|Q_{\varphi}\right\| \leq c_{0}, \quad\|\mathcal{F}(\varphi)\|_{\mathcal{C}^{0, \alpha}(\overline{\mathbb{D}})}<\delta,
$$

there exists a J-holomorphic disc $u \in \mathcal{C}^{1, \alpha}(\overline{\mathbb{D}})$ such that

$$
\|u-\varphi\|_{\mathcal{C}^{1, \alpha}(\overline{\mathbb{D}})} \leq 2 c_{0}\|\mathcal{F}(\varphi)\|_{\mathcal{C}^{0, \alpha}(\overline{\mathbb{D}})} .
$$


Proof. The key step is to prove that the derivative of $\mathcal{F}$ is locally Lipschitz under present assumptions. That is, there exists $c>0$ such that

$$
\left\|d_{\tilde{\varphi}} \mathcal{F}-d_{\varphi} \mathcal{F}\right\| \leq c\|\tilde{\varphi}-\varphi\|_{\mathcal{C}^{1, \alpha}(\overline{\mathbb{D}})}
$$

for any $\tilde{\varphi} \in \mathcal{C}^{1, \alpha}(\overline{\mathbb{D}})$ in a $\mathcal{C}^{1, \alpha}$-neighborhood of $\varphi$, say $\|\tilde{\varphi}-\varphi\|_{\mathcal{C}^{1, \alpha}(\overline{\mathbb{D}})}<1$. If such a statement is valid then one can simply set

$$
\eta=\min \left\{1, \frac{1}{2 c c_{0}}\right\} \quad \text { and } \quad \delta=\frac{\eta}{4 c_{0}}
$$

and apply the Theorem 2 to $X=\mathcal{C}^{1, \alpha}(\overline{\mathbb{D}}), Y=\mathcal{C}^{0, \alpha}(\overline{\mathbb{D}})$ and $x_{0}=\varphi$.

Let $h \in \mathcal{C}^{1, \alpha}(\overline{\mathbb{D}})$ and let $\|\varphi\|_{\mathcal{C}^{1, \alpha}(\overline{\mathbb{D}})}<c_{0}$. We need to prove that

$$
\left\|d_{\tilde{\varphi}} \mathcal{F}(h)-d_{\varphi} \mathcal{F}(h)\right\|_{\mathcal{C}^{0, \alpha}(\overline{\mathbb{D}})} \leq c\|\tilde{\varphi}-\varphi\|_{\mathcal{C}^{1, \alpha}(\overline{\mathbb{D}})}\|h\|_{\mathcal{C}^{1, \alpha}(\overline{\mathbb{D}})} .
$$

Note that

$$
d_{\varphi} \mathcal{F}(h)=h_{\bar{\zeta}}+A(\varphi) \overline{h_{\zeta}}+d_{\varphi} A(h) \overline{\varphi_{\zeta}},
$$

where $d_{\varphi} A(h)=\sum_{j=1}^{n} \frac{\partial A}{\partial z_{j}}(\varphi) h_{j}+\frac{\partial A}{\partial \bar{z}_{j}}(\varphi) \bar{h}_{j}$. We write

$$
d_{\tilde{\varphi}} \mathcal{F}(h)-d_{\varphi} \mathcal{F}(h)=I+I I+I I I,
$$

where

$$
\left\{\begin{array}{l}
I=(A(\tilde{\varphi})-A(\varphi)) \overline{h_{\zeta}} \\
I I=\left(d_{\tilde{\varphi}} A-d_{\varphi} A\right)(h) \tilde{\varphi}_{\zeta} \\
I I I=d_{\varphi} A(h)\left(\tilde{\varphi}_{\zeta}-\varphi_{\zeta}\right) .
\end{array}\right.
$$

Let us estimate each of the three parts.

Since $\mathbb{D}$ is convex and bounded the following embeddings are compact

$$
\mathcal{C}^{1, \alpha}(\overline{\mathbb{D}}) \subset \mathcal{C}^{1}(\overline{\mathbb{D}}) \subset \mathcal{C}^{0, \alpha}(\overline{\mathbb{D}})
$$

Therefore, one can bound the $\mathcal{C}^{0, \alpha}$-norm of the entries in $A(\tilde{\varphi})-A(\varphi)$ by their $\mathcal{C}^{1}$-norm. This implies existence of a constant $c_{1}>0$ depending on $\|\tilde{\varphi}\|_{\mathcal{C}^{1}(\overline{\mathbb{D}})}<1+c_{0}$ such that

$$
\|I\|_{\mathcal{C}^{0, \alpha}(\overline{\mathbb{D}})} \leq c_{1}\|\tilde{\varphi}-\varphi\|_{\mathcal{C}^{1, \alpha}(\overline{\mathbb{D}})}\|h\|_{\mathcal{C}^{1, \alpha}(\overline{\mathbb{D}})} .
$$

By a similar argument for $\frac{\partial A}{\partial z_{j}}(\tilde{\varphi})-\frac{\partial A}{\partial z_{j}}(\varphi)$ and $\frac{\partial A}{\partial \bar{z}_{j}}(\tilde{\varphi})-\frac{\partial A}{\partial \bar{z}_{j}}(\varphi)$ we get

$$
\|I I\|_{\mathcal{C}^{0, \alpha}(\overline{\mathbb{D}}} \leq c_{0} c_{2}\|\tilde{\varphi}-\varphi\|_{\mathcal{C}^{1, \alpha}(\overline{\mathbb{D}})}\|h\|_{\mathcal{C}^{1, \alpha}(\overline{\mathbb{D}})} .
$$

Finally, we have

$$
\|I I I\|_{\mathcal{C}^{0, \alpha}(\overline{\mathbb{D}})} \leq c_{3}\|\tilde{\varphi}-\varphi\|_{\mathcal{C}^{1, \alpha}(\overline{\mathbb{D}})}\|h\|_{\mathcal{C}^{1, \alpha}(\overline{\mathbb{D}})},
$$

where $c_{3}>0$ depends on the $\mathcal{C}^{0, \alpha}$-norm evaluated on the coefficients of $\frac{\partial A}{\partial z_{j}}(\varphi)$ and $\frac{\partial A}{\partial \bar{z}_{j}}(\varphi)$. The latter may be bounded by a constant times $c_{0}$. 
As stated above we will apply this approximation result to a shrinking family of sets in $\mathbb{D}$. Let $\left\{\Omega_{m}\right\}_{m \in \mathbb{N}}$ be such a family. Throughout the rest of this section we use the following convention: Given $\varphi \in \mathcal{C}^{1, \alpha}(\overline{\mathbb{D}})$ we denote by $\varphi_{m}$ its restriction to the set $\Omega_{m}$ and by $\mathcal{F}_{m}$ the corresponding operator defined as in (4) but mapping from $\mathcal{C}^{1, \alpha}\left(\bar{\Omega}_{m}\right)$ to $\mathcal{C}^{0, \alpha}\left(\bar{\Omega}_{m}\right)$.

Proposition 4. Let $\left\{\Omega_{m}\right\}_{m \in \mathbb{N}}$ be a shrinking family of sets in $\mathbb{D}$ and let $\varphi \in \mathcal{C}^{1, \alpha}(\overline{\mathbb{D}})$ be a map satisfying the limit condition

$$
\lim _{m \rightarrow \infty}\left\|\mathcal{F}_{m}\left(\varphi_{m}\right)\right\|_{\mathcal{C}^{0, \alpha}\left(\bar{\Omega}_{m}\right)}=0 .
$$

Then for every $m \in \mathbb{N}$ large enough, there exists a J-holomorphic map $u_{m}: \Omega_{m} \rightarrow \mathbb{R}^{2 n}$ such that

$$
\lim _{m \rightarrow \infty}\left\|u_{m}-\varphi_{m}\right\|_{\mathcal{C}^{1, \alpha}\left(\bar{\Omega}_{m}\right)}=0 .
$$

Proof. We need to verify that the constants $c_{0}>0$ and $\delta>0$ in Theorem 2 can be chosen independently of the sets $\Omega_{m}$. For this, we need two bounded linear extension operators $E_{k}: \mathcal{C}^{j, \alpha}(\overline{\mathbb{D}}) \rightarrow \mathcal{C}^{j, \alpha}\left(\bar{\Omega}_{m}\right), j=0,1$ (see e.g. [14, Theorem 4, p.177]). The remarkable fact is that their norms can be bounded by a constant $K_{j} \geq 1$ that is independent of the sets $\Omega_{m}$.

For points in $\Omega_{m}$ and $h \in \mathcal{C}^{1, \alpha}(\overline{\mathbb{D}})$ we have $d_{\varphi_{m}} \mathcal{F}_{m}\left(h_{m}\right)=d_{\varphi} \mathcal{F}(h)$. Hence, one can construct a bounded right inverse $Q_{m}$ of $d_{\varphi_{m}} \mathcal{F}_{m}$ by using the right inverse $Q_{\varphi}$ of $d_{\varphi} \mathcal{F}$. Indeed, given $g_{m} \in \mathcal{C}^{0, \alpha}\left(\bar{\Omega}_{m}\right)$, we take its extension $g=E_{0}\left(g_{m}\right)$ and proclaim $h_{m}=Q_{\varphi_{m}} g_{m}$ to be the restriction of $h=Q_{\varphi} g$ to $\Omega_{m}$. If $\left\|Q_{\varphi}\right\|<c_{0}$ and $\|\varphi\|_{\mathcal{C}^{0, \alpha}(\overline{\mathbb{D}})}<c_{0}$, the following estimate is valid

$$
\left\|Q_{\varphi_{m}} g_{m}\right\|_{\mathcal{C}^{0, \alpha}\left(\bar{\Omega}_{m}\right)} \leq\left\|Q_{\varphi} g\right\|_{\mathcal{C}^{0, \alpha}(\overline{\mathbb{D}})} \leq c_{0} K_{0} \cdot\left\|g_{m}\right\|_{\mathcal{C}^{0, \alpha}\left(\bar{\Omega}_{m}\right)}
$$

Moreover, $\left\|\varphi_{m}\right\|_{\mathcal{C}^{1, \alpha}\left(\bar{\Omega}_{m}\right)}<c_{0} \leq c_{0} K_{0}$.

Further, given $m \in \mathbb{N}$ let $\varphi_{m}, \tilde{\varphi}_{m} \in \mathcal{C}^{1, \alpha}\left(\bar{\Omega}_{m}\right)$ be such that

$$
\left\|\tilde{\varphi}_{m}-\varphi_{m}\right\|_{\mathcal{C}^{1, \alpha}\left(\bar{\Omega}_{m}\right)}<\frac{1}{K_{1}} \text {. }
$$

For $\varphi=E_{1}\left(\varphi_{m}\right)$ and $\tilde{\varphi}=E_{1}\left(\tilde{\varphi}_{m}\right)$ we have $\|\tilde{\varphi}-\varphi\|_{\mathcal{C}^{1, \alpha}(\overline{\mathbb{D}})}<1$. Hence as in (5) we can conclude that

$$
\left\|d_{\tilde{\varphi}_{m}} \mathcal{F}_{m}(h)-d_{\varphi_{m}} \mathcal{F}_{m}(h)\right\|_{\mathcal{C}^{0, \alpha}\left(\bar{\Omega}_{m}\right)} \leq c K_{1}^{2}\left\|\tilde{\varphi}_{m}-\varphi_{m}\right\|_{\mathcal{C}^{1, \alpha}\left(\bar{\Omega}_{m}\right)}\|h\|_{\mathcal{C}^{1, \alpha}\left(\bar{\Omega}_{m}\right)} .
$$

We now get the desired result from Theorem 2 by setting

$$
\eta=\min \left\{\frac{1}{K_{1}}, \frac{1}{2 c c_{0} K_{0} K_{1}^{2}}\right\} \text { and } \delta=\frac{\eta}{4 c_{0} K_{0}} .
$$


We now prove a local approximation statement that will be used in the proof of the main theorem. We mimic the proof of [3, Lemma 3.5].

Theorem 5. Let $J \in \mathcal{J}$. Given $\epsilon>0$, a smoothly embedded arc $\Gamma \subset \mathbb{C}$ and a $\mathcal{C}^{2}$-map $\varphi: \Gamma \rightarrow \mathbb{R}^{2 n}$, there exists a neighborhood $U$ of $\Gamma$ and a $J$ holomorphic map $u: U \rightarrow \mathbb{R}^{2 n}$ such that $\|u-\varphi\|_{\mathcal{C}^{1, \alpha}(\Gamma)}<\epsilon$.

Proof. Without loss of generality we can assume that $\Gamma \subset \mathbb{D} \cap \mathbb{R}$. By (3) the $J$-holomorphicity condition equals $u_{y}=J(u) u_{x}$. Hence, we may extend $\varphi$ to a function that is quadratic in $y$ and whose $\bar{\partial}_{J}$-derivative vanishes up to the first order along $\Gamma$. In particular, for

$$
\Omega_{m}=\left\{z \in \mathbb{C}: \operatorname{dist}(z, \Gamma)<\frac{1}{m}\right\}
$$

there exist $m_{0}>0$ and $C_{\alpha}>0$ such that $\Omega_{m} \subset \mathbb{D}$ and

$$
\left\|\left(\varphi_{m}\right)_{\bar{\zeta}}+A\left(\varphi_{m}\right) \overline{\left(\varphi_{m}\right)_{\zeta}}\right\|_{\mathcal{C}^{0, \alpha}\left(\bar{\Omega}_{m}\right)}<\frac{C_{\alpha}}{m}
$$

for every $m \geq m_{0}$. The rest follows from the Proposition 4 above.

\section{The Runge-Type approximation}

As mentioned in the introduction, our result is an application of the following Runge-type theorem provided by A. Gournay [5].

Theorem 6. Let $M$ be a smooth compact manifold equipped with a regular almost complex structure and admitting a doubly tangent property. Suppose we are given $\epsilon>0$, a compact Riemann surface $\Sigma$, an open set $U \subset \Sigma$, a $J$-holomorphic map $\varphi: U \rightarrow M$ and a compact set $K \subset U$. Then, provided that there is a $\mathcal{C}^{0}$ extension of $\varphi$ to $\Sigma$, there exists a J-holomorphic map $u: \Sigma \rightarrow M$ such that $\|u-\varphi\|_{\mathcal{C}^{0}(K)}<\epsilon$.

We include below a brief discussion on the proof in order to explain why the statement can be applied in the present case.

Provided that there are no topological obstructions, one can define a new map extending the initial data $\left.\varphi\right|_{K}$ to $\Sigma$ in a $\mathcal{C}^{\infty}$-fashion. Let us denote it by $\varphi$ again. Of course such a map needs not to be holomorphic on $\Sigma \backslash K$ and one can express this locally. Fix $\zeta_{0} \in \Sigma \backslash K$ and the following two charts: a chart $\psi$ on $\Omega_{0} \subset \Sigma$ with $\psi\left(\zeta_{0}\right)=0$ and a chart $\phi$ on $M$ taking $q=\varphi\left(\zeta_{0}\right) \in M$ to $0 \in \mathbb{R}^{2 n}$ and satisfying $\phi^{*}(J)(0)=J_{\text {st }}$. There exist $a, b \in \mathbb{R}^{2 n}$ such that

$$
\psi \circ \varphi \circ \phi^{-1}(z)=a z+b \bar{z}+O\left(|z|^{2}\right),
$$

where $b \neq 0$ is equivalent to $\bar{\partial}_{J} \varphi \neq 0$. 
First of the two key assumptions in the method of A. Gournay is that the manifold enjoys the doubly tangent property. That is, for almost every $q \in M$ and almost every pair $a, b \in \mathbb{R}^{2 n}$, there exists a $J$-holomorphic sphere $H_{a, b}^{r}: \mathbb{C} P^{1} \rightarrow M$ whose local (Laurent) expansion equals to

$$
\psi \circ H_{a, b}^{r} \circ \phi^{-1}(z)=a z+b r^{2} / z+O\left(r^{1+\epsilon}\right) .
$$

Here, $\epsilon>0$ and $r>0$ are such that for $\frac{r}{\left(1+r^{\epsilon}\right)}<|z|<r\left(1+r^{\epsilon}\right)$ we have $\psi^{-1}(z) \in \Omega_{0}$. Hence, using an appropriate cut-off function, the map $\varphi$ may be replaced by $H_{a, b}^{r}$ in the vicinity of $\zeta_{0}$. Moreover, since $H_{a, b}^{r}$ is holomorphic and almost agrees with $\varphi$ on $|z|=r$, such a surgery diminishes the 'size' of the $\bar{\partial}_{J}$-derivative. The author calls such a procedure grafting and he repeats it finitely many times until reaching the desired bounds (see [5, §3.1]).

Once an approximate solution is constructed (we denote it by $\varphi: \Sigma \rightarrow M$ again), the $\bar{\partial}_{J}$-equation can be solved similarly as in our $\S 1$. Let us briefly explain this. The non-linear $\bar{\partial}_{J}$-operator, now defined globally, may be linearized at a compact curve $u$ so that the corresponding Fredholm operator $D_{u}$ maps from $\mathcal{C}^{\infty}\left(\Sigma, \varphi^{*} T M\right)$ to $\mathcal{C}^{\infty}\left(\Sigma, \Lambda^{0,1} \varphi^{*} T M\right)$ (see [9, (3.1.4.)]). The notion of regularity refers to its surjectivity. In particular, for us the structure $J$ is regular when $D_{u}$ is onto for every $J$-holomorphic sphere. The idea is to find a bounded right inverse for $D_{\varphi}$. That is, given $\eta \in \mathcal{C}^{\infty}\left(\Sigma, \Lambda^{0,1} \varphi^{*} T M\right)$ we seek solution $\xi \in \mathcal{C}^{\infty}\left(\Sigma, \varphi^{*} T M\right)$ of $D_{\varphi} \xi=\eta$ with bounds.

In the cited paper the latter is obtained in two steps. Firstly, it is shown in [5, §3.2] that it suffices to solve local equations $D_{\varphi_{j}} \xi_{j}=\eta_{j}$, where $\left\{\left(\varphi_{j}, \xi_{j}, \eta_{j}\right)\right\}_{j \geq 0}$ stands for slightly perturbed data $(\varphi, \xi, \eta)$ restricted either to the original surface $\Sigma_{0}=\Sigma$ or to one of the finitely many grafts $\Sigma_{j}=\mathbb{C} P^{1}$, $j>0$. Secondly, it is proved in [5, §3.3] that though the local equations for $j>0$ interact with the one for $j=0$ the iteration starting at $\xi_{0}=0$ is indeed contractible. Here the regularity of the structure is crucial since it ensures that the local equation is always solvable along the grafts. In contrast, the inversion of the linear equation for $j=0$, is very subtle [5, §3.3].

Finally, it is worth mentioning that the norms in question are not the ones associated with Sobolev or Hölder spaces. The reason lies in the fact that each graft increases the $L^{p}$ norm of $d \varphi$ by a quantity that is a priori significant. Furthermore, the number of surgeries is not bounded in general. Hence, the local Lipschitz constant grows with $\partial \varphi$ when $D_{\varphi}$ is treated as a map from $W^{1, p}\left(\Sigma, \Lambda^{0,1} \varphi^{*} T M\right)$ to $L^{p}\left(\Sigma, \varphi^{*} T M\right), p>2$. This makes it impossible to use the Implicit function theorem. Hence a certain sup-norm introduced by C. Taubes [16] is used (see also [4, §4]). 
We now state the corollary that will be used in the proof of Theorem 1 .

Corollary 7. Let $(M, J)$ be as in Theorem 6 . Let $K \subset \partial \mathbb{D}$ be a compact set and let the map $\varphi$ be continuous near $\overline{\mathbb{D}}$ and $J$-holomorphic near $K \cup\{0\}$. Given $\epsilon>0$, there exists $u \in \mathcal{O}(\overline{\mathbb{D}}, M, \varphi(0))$ such that $\|u-\varphi\|_{\mathcal{C}^{0}(K)}<\epsilon$.

Proof. First note that the map $\varphi$ can be continuously extended to $\Sigma=\mathbb{C} P^{1}$. Hence, the Runge-type theorem guarantees existence of a $J$-holomorphic map approximating $\varphi$ on $K \cup\{0\}$. It remains to explain why the above proof can be adopted slightly in order to obtain $u(0)=\varphi(0)$.

The simplest way to do this is by adding an 'un-necessary graft' at the center. That is, we start by replacing the map $\varphi$ with an appropriate graft $H_{a, b}^{r}$ near $0 \in \mathbb{D}$. Since $\bar{\partial}_{J} \varphi(0)=0$ we have $b=0$ and $\varphi(0)=H_{a, b}^{r}(0)$ here. We index this graft with $j=1$ and then proceed with the usual grafting procedure for $j>1$. Moreover, we add a point-wise restriction $\xi(0)=0$ each time when solving the local linear equation $D_{\varphi_{1}} \xi_{1}=\eta_{1}$. Since $J$ is regular this does not object the surjectivity of $D_{\varphi_{1}}$. Indeed, check [9, §3.4]. Hence, all the key estimates remain fulfilled. In particular, [5, Corollary 2.5.5.] can be used in the iterative scheme from [5, §3.3].

\section{Proof of Theorem 1}

The direct construction of a Poletsky disc follows from Theorem 5 and Corollary 7. Indeed, as in [13], we prove the following stronger statement.

Theorem 8. Let $(M, J)$ be as in Theorem 1 and equipped with some Riemannian metric. Given a point $p \in M$, a positive constant $\epsilon>0$ and $a$ $\mathcal{C}^{2}$-map $\lambda: \partial \mathbb{D} \rightarrow M$, there exist a disc $u \in \mathcal{O}(\overline{\mathbb{D}}, M, p)$ and a set $E \subset[0,2 \pi)$ such that $|E|<\epsilon$ and $\operatorname{dist}\left(u\left(e^{i t}\right), \lambda\left(e^{i t}\right)\right)<\epsilon$ for $t \in[0,2 \pi) \backslash E$.

Proof. As pointed out above the direct method consists of two steps. We first make a piece-wise holomorphic approximation of $\lambda$. Then we use the Runge-type theorem to extend this map to the whole disc. The second step can be understood as adding finitely many poles (grafts).

Fix $e^{i t} \in \partial \mathbb{D}$. Let $\phi_{t}: V_{t} \rightarrow \mathbb{B}$ be a local chart mapping a neighborhood of $\lambda\left(e^{i t}\right)$ into a neighborhood of the origin in $\mathbb{R}^{2 n}$ and satisfying $\phi_{t}^{*}(J) \in \mathcal{J}$. We define $\Gamma_{t}^{\prime} \subset \partial \mathbb{D}$ to be the largest connected subarc including $e^{i t}$ and satisfying $\lambda\left(\Gamma_{t}^{\prime}\right) \subset \lambda(\partial \mathbb{D}) \cap V_{t}$. By compactness, there are points $t_{1}, \ldots, t_{k} \in[0,2 \pi)$ such that the the union $\cup_{j=1}^{k} \Gamma_{t_{j}}^{\prime}$ covers the whole $\partial \mathbb{D}$. Moreover, we can choose smaller pairwise disjoint subarcs $\Gamma_{t_{j}} \subset \subset \Gamma_{t_{j}}^{\prime}$ satisfying

$$
\left|\partial \mathbb{D} \backslash \cup_{j=1}^{k} \Gamma_{t_{j}}\right|<\epsilon .
$$


By Theorem 5 there exist $J$-holomorphic maps $u_{j}: U_{j} \rightarrow V_{z_{j}}$ that are defined on pairwise disjoint neighborhoods and $\mathcal{C}^{1, \alpha}$-close to $\phi_{j} \circ \lambda$ on $\Gamma_{t_{j}}$. Moreover, by the classical Nijenhuis-Woolf theorem [10] there exists a small $J$-holomorphic disc $u_{0}$ centered at $p$. Since $M$ is connected we can join these pieces into a continuous map $\varphi$ defined on a neighborhood of $\overline{\mathbb{D}}$ and satisfying $\varphi(0)=u_{0}(0)=p$. The rest follows from the Corollary 7 applied to the compact set $K=\cup_{j=1}^{k} \Gamma_{t_{j}}$.

The research of the author was supported in part by the research program P1-0291 and the grant J1-7256 from ARRS, Republic of Slovenia. A large part of the result was created during his stay at the University of Oslo, Spring 2017. He wants to thank prof. Erlend F. Wold for his hospitality. He also wants to thank prof. Barbara Drinovec-Drnovšek for her useful remarks on an earlier version of the paper.

\section{REFERENCES}

[1] F. Bertrand and U. Kuzman Local approximation of non-holomorphic maps in almost complex manifolds. J. Math. Anal. Appl., to appear. arXiv:1704.01781

[2] B. Drinovec DrnovšEk and F. Forstnerič Characterizations of projective hulls by analytic discs. Illinois J. Math. 56 (2013), 53-65.

[3] D. Chakrabarti Coordinate neighborhoods of arcs and the approximation of maps into (almost) complex manifolds. Michigan Math. J. 55 (2007), 299-333.

[4] S. K. Donaldson The approximation of instantons. Geom. Funct. Anal. 3, (1993), 179-200.

[5] A. Gournay A Runge approximation theorem for pseudo-holomorphic maps. Geom. Funct. Anal. 22 (2012), 311-351.

[6] U. Kuzman, Poletsky theory of discs in almost complex manifolds. Comp. Var. Ell. Equat., 59 (2014), 262-270.

[7] F. Larusson and R. Sigurdsson, Plurisubharmonic functions and analytic discs on manifolds, J. Reine. Angew. Math., 501 (1998), 1-39.

[8] B. S. Magnusson, Analytic discs, global extremal functions and projective hulls in projective space. Illinois J. Math. 58 (2014), 391-404.

[9] D. McDuff and D. SAlamon, J-holomorphic curves and symplectic topology, second edition. American Mathematical Society Colloquium Publications, 52. American Mathematical Society, Providence, RI, 2012. xiv+726 pp.

[10] A. Nijenhuis and W. Woolf, Some integration problems in almost-complex and complex manifolds. Ann. Math. 77(1963), 429-484.

[11] E. A. Poletsky, Plurisubharmonic functions as solutions of variational problems, In: Several complex variables and complex geometry, Part 1 (Santa Cruz, CA, 1989), 163-171, Proc. Sympos. Pure Math., 52, Part 1, Amer. Math. Soc., Providence, RI, 1991. 
[12] J.-P. Rosay, Poletsky theory of disks on holomorphic manifolds, Indiana Univ. Math. J., 52 (2003), 157-169.

[13] J.-P. Rosay, Discs in complex manifolds with no bounded plurisubharmonic functions. Proc. Amer. Math. Soc. 132 (2004), 2315-2319.

[14] E. M. Stein, Singular integrals and differentiability properties of functions. Princeton Math. Ser.30, Pergamon Univ. Press, Princeton, NJ, 1962.

[15] A. Sukhov and A. Tumanov, Deformations and transversality of pseudoholomorphic discs, J. d'Analyse Math., 116 (2012), 1-16.

[16] C.-H. Taubes The existence of anti-self-dual conformal structures. J. Differential Geom. 36, (1992), 163-253. 